\title{
Chirality modifies the interaction between knots
}

\author{
Saeed Najafi, ${ }^{1}$ Luca Tubiana, ${ }^{2}$ Rudolf Podgornik, ${ }^{3}$ and Raffaello Potestio ${ }^{1, *}$ \\ ${ }^{1}$ Max Planck Institute for Polymer Research, Ackermannweg 10, 55128 Mainz, Germany \\ ${ }^{2}$ University of Vienna, Computational Physics Group, Sensengasse 8, 1090, Vienna, Austria \\ ${ }^{3}$ University of Ljubljana, Faculty for Mathematics and Physics, Jadranska 19, 1000, Ljubljana
}

(Dated: February 23, 2018)

\begin{abstract}
In this study we consider an idealization of a typical optical tweezers experiment involving a semiflexible double-knotted polymer, with steric hindrance and persistence length matching those of dsDNA in high salt concentration, under strong stretching. Using exhaustive Molecular Dynamics simulations we show that not only does a double-knotted dsDNA filament under tension possess a free energy minimum when the two knots are intertwined, but also that the depth of this minimum depends on the relative chirality of the two knots. We rationalize this dependence of the effective interaction on the chirality in terms of a competition between chain entropy and bending energy.
\end{abstract}

The study of physical knots in polymers is an important emerging topic in biophysics and soft matter in general. Since the original conjecture that knots should be ubiquitous in sufficiently long chains [1, 2], later proved by Sumners and Whittington [3], knots have been found or tied in a variety of biopolymers, from DNA [4 $[6]$ to proteins [7-13] and even actin filaments [14], and have been shown to have a large impact on the biological function of proteins and DNA [15, 16]. More recently, several studies have shed light on the relevance of knots also in nanotechnological applications [14, 17,20].

Physical knots appear and diffuse spontaneously along polymer chains 21 26], on which they acquire a metastable tightness [27 29], and can affect structural and dynamical properties like their radius of gyration 30 33], tensile strength [14, 34], diffusion constants 35, 36], and translocation dynamics through a pore [19].

Most studies up to now focused on the properties of single knots, investigating how knot occurrence probability [52, 53], size [33, 39, 40], and dynamical properties depend on physical characteristics of the system under study, such as polymer thickness 50,54 , confinement [5, 6, 18, 55, 56], stretching force [24, 26, 38, 47], crowding and solution density [57 59]. Polymers, though, can host multiple knots. These, referred to as composite knots, are actually the most probable type of knots in the case of long polymers [3, 37]. The study of composite knots is of great interest since the presence of interactions among their prime components may alter the overall properties of the polymer. For example, knot colocalization on a stretched polymer may significantly diminish its tensile strength with respect to a chain with a series of localized, non-intertwined prime knots.

Since prime knots tied on polymers in solutions or under weak mechanical stretching are weakly localized, their length growing sublinearly with the length of the polymer 33, 38 40, 47, when tied on the same polymer they are expected, in the thermodynamic limit of

\footnotetext{
* potestio@mpip-mainz.mpg.de
}

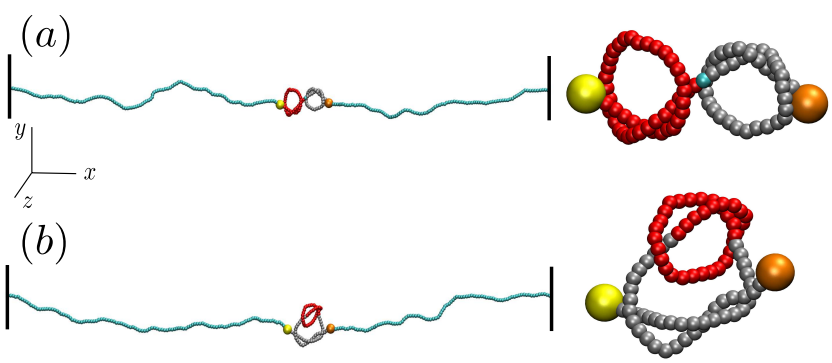

FIG. 1. Snapshots from simulations of a chain with bending rigidity $\kappa=20 k_{B} T$ containing two $3{ }_{1}$ knots of different chirality. The shortest knotted portions of isolated prime components (zoomed in the right part of the figure) are highlighted in red and grey. Yellow and orange beads, whose sizes were artificially increased for clarity, mark the beginning and end of the composite knots, respectively.

infinitely long chains, to be statistically independent. In fact, computational studies have shown that in the thermodynamic limit the characteristic exponent, relating the configurational entropy of a polymer ring to its contour length, can be written as in the case of an unknotted ring augmented by the number of knots that are present, and that the knotting probability of composite knots factorizes into that of their prime knot components [41, 42]. However, in finite-size polymers the situation can be quite different. In this case the size of knots is non-negligible and therefore they can not be mapped onto independent point-like decorations [43]. On the contrary, knots can be expected to be intertwined, so that one is inside another, and to interact with each other. Dommersnes et al. have shown that knots tied on a short unscreened charged ring become as tight as possible and maximize their relative distances along the ring [44], while Virnau and coworkers, simulating a double-knotted stretched dsDNA chain, have shown that knots can become intertwined in a minimum free energy configuration [45]. Furthermore, a recent study by one of us [46] reported that knot size remains an important factor in freely jointed rings of up to thousands of bonds, where the probability of finding intertwined knots remains sig- 
nificant. These results suggest that finite size effects are relevant in most biological phenomena and nanotechnological applications involving stiff polymers such as dsDNA, and that indeed the overall effect of knots may depend on some complex interaction between them.

To shed further light on the interactions between knots along a finite-size polymer, we consider an idealization of a typical optical tweezers experiment [38, 45, 47] in which a semiflexible double-knotted chain is stretched between two impenetrable walls, describing the effect of tweezer confinement on the terminal polystyrene beads (see Fig. 1). The separation of the chain termini is such that the knotted polymer is in the high-force stretching regime in which knots are strongly localized, their size showing only small fluctuations around an average size which scales solely with the applied force [38, 47]. Using exhaustive Molecular Dynamics (MD) simulations of polymers containing different pairs of prime knots, we show that not only does a double-knotted dsDNA filament under tension possess a free energy minimum when the two knots are intertwined [45], but also that the depth of this minimum depends on the relative chirality of the two knots. We show that this dependence of the effective interaction on the chirality originates from an interplay between chain entropy and bending energy, which is dominated by the latter.

The dsDNA is modeled as a chain of $N=300$ beads of diameter $\sigma$ connected by FENE (finitely extensible nonlinear elastic) bonds [48, 49]. A Weeks-ChandlerAnderson (WCA) potential enforces the excluded volume, and a harmonic bending energy term induces a finite persistence length. The chain is stretched along the $X$ axis, and its termini are kept fixed in correspondence of two repulsive walls, modeled as WCA potentials, which prevent the knots from untying. The total potential energy of the system is thus:

$$
U_{\text {tot }}=U_{\mathrm{WCA}}+U_{\mathrm{FENE}}+U_{\text {bending }}+U_{\text {walls }}
$$

where the WCA potential is taken as:

$$
\begin{aligned}
& U_{\mathrm{WCA}}=\frac{1}{2} \sum_{(i, j), j \neq i}^{N} V\left(d_{i, j}\right) \\
& V(r)=\left\{\begin{array}{l}
4 \epsilon\left[\left(\frac{\sigma}{r}\right)^{12}-\left(\frac{\sigma}{r}\right)^{6}+\frac{1}{4}\right] \text { for } r \leq 2^{1 / 6} \sigma . \\
0 \text { otherwise }
\end{array}\right.
\end{aligned}
$$

The WCA strength $\epsilon=1 k_{B} T$ and the characteristic length scale $\sigma$ are taken as the energy and length units, respectively. All other dimensional quantities are expressed in terms of reduced units defined through $\epsilon, \sigma$ and the bead unit mass $m$. Time is measured in the MD time units $\tau_{M D}=\sigma \sqrt{m / \epsilon}=1$. The FENE potential reads:

$$
U_{\mathrm{FENE}}=-\sum_{i=0}^{N-2} \frac{\kappa_{\text {fene }}}{2}\left(\frac{R_{0}}{\sigma}\right)^{2} \ln \left[1-\left(\frac{\left|\vec{u}_{i}\right|}{R_{0}}\right)^{2}\right]
$$

where $\vec{u}_{i} \equiv \vec{r}_{i+1}-\vec{r}_{i}$ is the vector pointing to the bead $i+1$ from bead $i$, and $\left|\vec{u}_{i}\right|$ is thus the distance of the bead centers $i$ and $i+1$. The values of the maximum bond length $R_{0}=1.5 \sigma$ and the FENE interaction strength $\kappa_{\text {fene }}=30 \epsilon$ are the customary ones for the Kremer-Grest model [48]. The harmonic bending potential is taken in its standard form:

$$
U_{\text {bending }}=\sum_{i=1}^{N-1} \kappa\left(1-\frac{\vec{u}_{i} \cdot \vec{u}_{i+1}}{\left|\vec{u}_{i}\right|\left|\vec{u}_{i+1}\right|}\right)
$$

where $\kappa=20 k_{B} T$ is the bending stiffness of the chain, inducing a persistence length $l_{p}=20 \sigma$. Setting $\sigma=$ $2.5 \mathrm{~nm}$ gives us $l_{p}=50 \mathrm{~nm}$, typical of dsDNA in high monovalent salt concentrations [50].

The potential of Eq. 1 is used to perform underdamped MD simulations in an implicit solvent with a Langevin thermostat and time step $\Delta t=0.01 \tau_{M D}$, with the friction self-correlation time $\tau_{\text {frict }}=10^{3} \tau_{M D}$.

To assign a topological state to subsections of the chain, we used the Minimally Interfering Closure [51]. We define a knotted portion of the chain as the shortest segment featuring a specific knotted topology upon closure according to the Alexander polynomial [61]. With some abuse of language, we will refer to such portions as "knots" in what follows. By applying this procedure to composite knots, we are able to identify both the chain portion hosting the whole composite knot, as well as those hosting its "isolated" prime components. Following ref. [46], we consider a prime component to be isolated when it can be excised, and its ends joined, without at the same time untying the second knot, see Fig 1 .

Six topologically different setups have been investigated, namely: $\left(3_{1}^{+} 3_{1}^{+}\right),\left(3_{1}^{+} 3_{1}^{-}\right),\left(4_{1} 3_{1}^{+}\right),\left(4_{1} 3_{1}^{-}\right),\left(5_{1}^{-} 3_{1}^{-}\right)$, $\left(5_{1}^{-} 3_{1}^{+}\right)$. The chirality of each prime knot has been established using the writhe, that is, the sum of the signed crossings of the knot in its reduced diagram. + and superscripts refer to right and left handedness according to the right-hand rule convention [17, 60]. In our setup, $(++)$ and $(--)$ composite knots are related by a mirror transformation; therefore, we do not attach any importance to the overall chirality of the composite knot, but only on the relative chirality of its prime components. Since no cross-passage is allowed in our simulations, the chirality of all knots is preserved during the simulations. In all setups, the termini of the chain were kept fixed at a distance of $L=205 \sigma$, corresponding to forces of about $1-4 \mathrm{pN}$ at $T=300 \mathrm{~K}$, depending on the knot complexity, applied on both termini. At these forces, corresponding to a strong stretching regime [47], the knot lengths show relatively small fluctuations around their average values, as reported in Table [. For each of the six topologies under examination, 40 independent simulations were performed, each consisting of an initial equilibration phase of $2 \times 10^{7} \tau_{M D}$ and a production phase of $2 \times 10^{9} \tau_{M D}$.

As a first case we investigate the $3_{1}^{ \pm} 5_{1}^{-}$system, taking into account two different chiralities of the trefoil knot: $3_{1}^{+}$and $3_{1}^{-}$. The fractions of intertwined states in the $3_{1}^{+}$ and $3_{1}^{-}$cases are 0.585 and 0.447 , respectively. In both setups the largest knot, the $5_{1}$, swells up to let the $3_{1}$ 


\begin{tabular}{|c|c|c|c|c|}
\hline & \multicolumn{2}{|c|}{ Separated } & \multicolumn{2}{c|}{ Intertwined } \\
\cline { 2 - 5 } & $l_{k}^{31}$ & $l_{k}^{\text {other }}$ & $l_{k}^{\text {2ncl }}$ & $l_{k}^{\text {comp }}$ \\
\hline $5_{1}^{-} \# 3_{1}^{-}$ & $29.5 \pm 3.1$ & $50.5 \pm 4.3$ & $30.3 \pm 4.7$ & $72.4 \pm 3.7$ \\
\hline $5_{1}^{-} \# 3_{1}^{+}$ & $29.6 \pm 3.1$ & $50.6 \pm 4.3$ & $33.9 \pm 5.2$ & $72.9 \pm 3.7$ \\
\hline $4_{1} \# 3_{1}^{+}$ & $32.9 \pm 3.6$ & $44.5 \pm 4.0$ & $30.4 \pm 3.3$ & $72.6 \pm 3.8$ \\
\hline $4_{1} \# 3_{1}^{-}$ & $32.9 \pm 3.7$ & $44.5 \pm 4.1$ & $30.4 \pm 3.3$ & $72.6 \pm 3.8$ \\
\hline $3_{1}^{+} \# 3_{1}^{+}$ & $35.5 \pm 2.2$ & & $33.0 \pm 3.7$ & $67.2 \pm 4.1$ \\
\hline $3_{1}^{+} \# 3_{1}^{-}$ & $35.7 \pm 2.3$ & & $34.7 \pm 3.9$ & $67.0 \pm 4.0$ \\
\hline
\end{tabular}

TABLE I. Average knot lengths for different topologies. In the "Separated" columns are reported the knot lengths for the prime components when they are not intertwined. The "Intertwined" columns report the average lengths of the isolated prime component that has been included (labeled $l_{k}^{\text {incl }}$ ) and of the whole composite knot in an intertwined configuration (labeled $\left.l_{k}^{\text {comp }}\right)$. In those cases where the $4_{1}$ is entwined by the $3_{1}$, constituting the $8 \%$ of the intertwined configurations for this topology, the size of the $4_{1}$ is $42.5 \pm 3.7$, and the length of the whole composite knot is $70.1 \pm 3.6$. We remark that the knots under investigation here are quite tight, with lengths almost half $(55 \%)$ those of the knots studied in ref. [45] in the case of the $3_{1} \# 4_{1}$ systems.

knot in. The frequency with which the trefoil enters or exits the $5_{1}$ is $7.6 \times 10^{-9} \tau_{M D}^{-1}$ for the $5_{1}^{-} 3_{1}^{-}(--)$pair, and $13.2 \times 10^{-9} \tau_{M D}^{-1}$ for the $5_{1}^{-} 3_{1}^{+}(-+)$pair 62 . The data in Table \ show that the length of separate prime components is independent of their relative chirality for all topologies under study. This allows us to introduce a collective descriptor, or an order parameter, $D$, defined as the oriented distance between the knot centers. This is measured as the number of chain beads from the center of the $5_{1}$ knot to the center of the $3_{1}$ knot, $D=c_{3_{1}}-c_{5_{1}}$. A similar definition can be provided for the configurations in which the two knots are intertwined and the knot identification algorithm allows us to identify only one prime component, the one which has been entwined by the other knot. In those cases we identify the center of the swollen knot with the center of the whole composite knot in the expression for $D$ [45]. Therefore, $D=0$ in those configurations in which the two knots are intertwined and the innermost knot is located exactly at the center of the outermost knot. A schematic representation of this collective descriptor is provided in Fig. 2.

By counting the relative number of MD configurations for which the knot components are separated by a given distance $D$ we can obtain the probability distribution $P(D)$ and, correspondingly, the free energy $F(D)=-k_{B} T \ln P(D)$. The latter is reported for the two $3{ }_{1}^{ \pm} 5_{1}^{-}$systems in Fig. 3 a.

From the profiles in Fig. 3a we observe that $F(D)$ increases with increasing $|D|$, a behaviour that can be intuitively attributed to the entropic cost of placing two knots at large distance on a long, yet finite chain [43]. Consistent also with previous observations [45], for small values of $|D|$ we detect two barriers and two minima in the free energy, corresponding to configurations in which the two knots are intertwined. Most interestingly and unexpectedly, we observe that the depth of these minima depends on the relative chirality of the knots. When the two components have opposite chirality, the corresponding free energy minimum is $\sim 1 k_{B} T$ deeper than for the system in which the two chiralities are identical.

This conclusion is reinforced by simulations performed

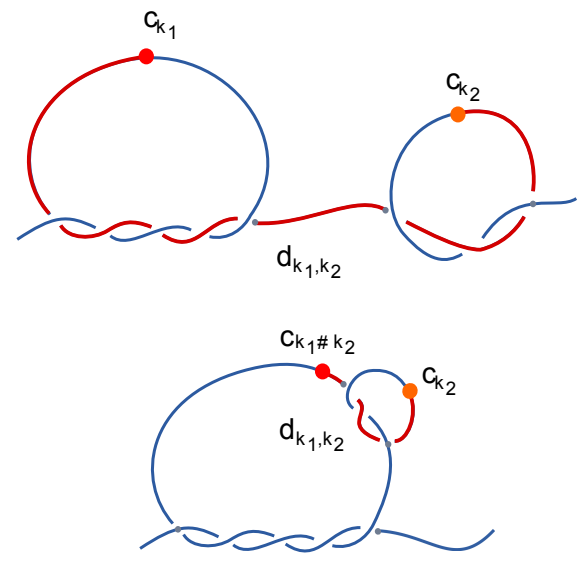

FIG. 2. Schematics of the collective order parameter $D$ measuring the linear distance between two prime knots, in this case a $5_{1}$ and a $3_{1}$. When both prime components are isolated, the order parameter is given by $D=c_{k_{2}}-c_{k_{1}}$, where $c_{k_{i}}=\left(e_{k_{i}}+s_{k_{i}}\right) / 2$ is the center of knot $i$ on the chain. Here $e_{k_{i}}$ and $s_{k_{i}}$ stand for the last and the first bead of the $i$-th isolated knotted portion. $k_{1}$ is always taken to be the most complex knot, in this case the $5_{1}$. When the knots are intertwined, we identify the center of the swollen knot with the center of the whole composite knot, $c_{k_{1} \# k_{2}}$.

on a $3_{1}^{ \pm} 4_{1}$ composite knot, where one of the knots, the $4_{1}$, is achiral. The free energy in this case, reported in Fig. $3 \mathrm{~b}$, does not depend on the chirality of the $3_{1}$ component, as it is indeed expected since there are no other chiral entities in the setup. In the simulations the intertwined states with the $4_{1}$ including the trefoil are the most probable, making for the $92 \%$ of observed configurations [63].

To understand if the sole relative chirality of two knots can mark a difference in their preference to stay intertwined or to separate along a chain under tension, we consider a system composed by two otherwise identical trefoil knots. In this case we use as order parameter the absolute value of $D$, since when the two trefoil knots have the same chirality they become effectively indistinguish- 


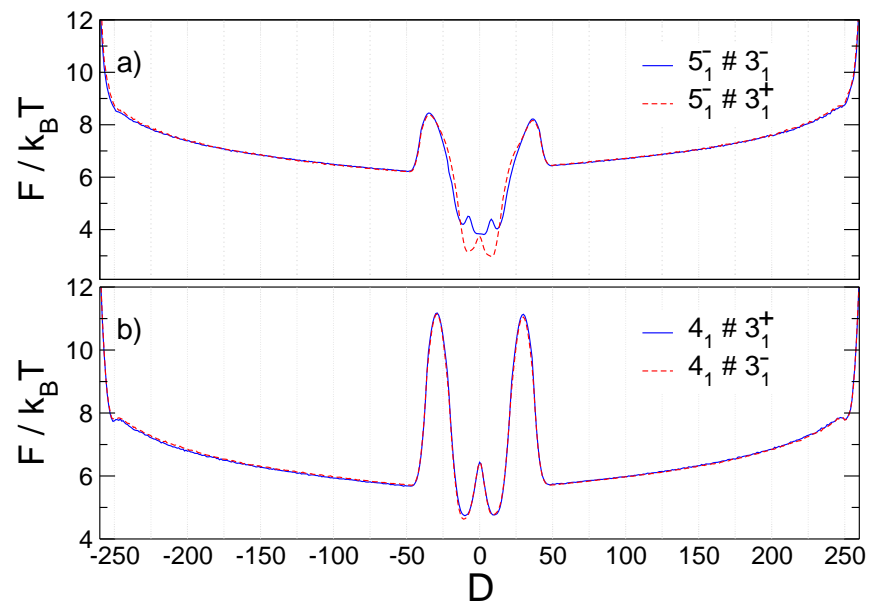

FIG. 3. (a) Free energy, $F(D)$, as a function of the linear distance between the knots for $5_{1}^{-} 3_{1}^{-}$(blue solid line) and $5_{1}^{-} 3_{1}^{+}$ (red dashed line). (b) Same as in (a) for the knots $4_{1} 3_{1}^{+}$(blue solid line) and $4_{1} 3_{1}^{-}$(red dashed line). Note that in this latter case the two quantities coincide. The free energies for the $4_{1} 3_{1}^{ \pm}$reported in panel (b) can be decomposed to distinguish the cases in which the $4_{1}$ intakes the $3_{1}$ in and those in which the opposite happens; no substantial difference depending on the relative chirality is to be observed (data not shown).

able. The free energy profiles, Fig. 4h, corroborate that also in this case the system with two knots having opposite chiralities has a lower free energy minimum when the two components are intertwined. We note here that the presence of the two repulsive walls may impact the free energy profiles, since our parameter $D$ does not distinguish the whether the knots are near the walls or far from them. In order to rule out possible distortions due to the interactions between knots and walls, the free energy profiles have been also computed excluding all those configurations in which the knots were separated from the wall by a distance lower than $2 l_{p}$. The resulting profiles (data not shown) are perfectly consistent with the ones reported in Figs. 3 and 4, thus validating the robustness of the observed behavior.

Further insight into the cause of this effect can be obtained by analyzing the size of the intertwined configurations. As reported in Table I] we find that for all investigated topologies the length of the composite knot in the intertwined state, $l_{\text {comp }}$, does not depend on the relative chirality of the prime components. Interestingly, we observe that both for the $3_{1}^{ \pm} 5_{1}^{-}$and for the $3_{1}^{ \pm} 3_{1}^{-}$systems the length of the nested, isolated prime components in the intertwined state is slightly larger in the $(+-)$ case [64.

We proceed to separate the free energy $F(D)$ in its energetic and entropic components, by first computing the average internal (potential) energy of the configurations, and subsequently obtaining the entropy through the standard relation $F=E_{\text {int }}-T S$. The results, reported in Fig. 4 a-c, show two interesting features. First, they con-

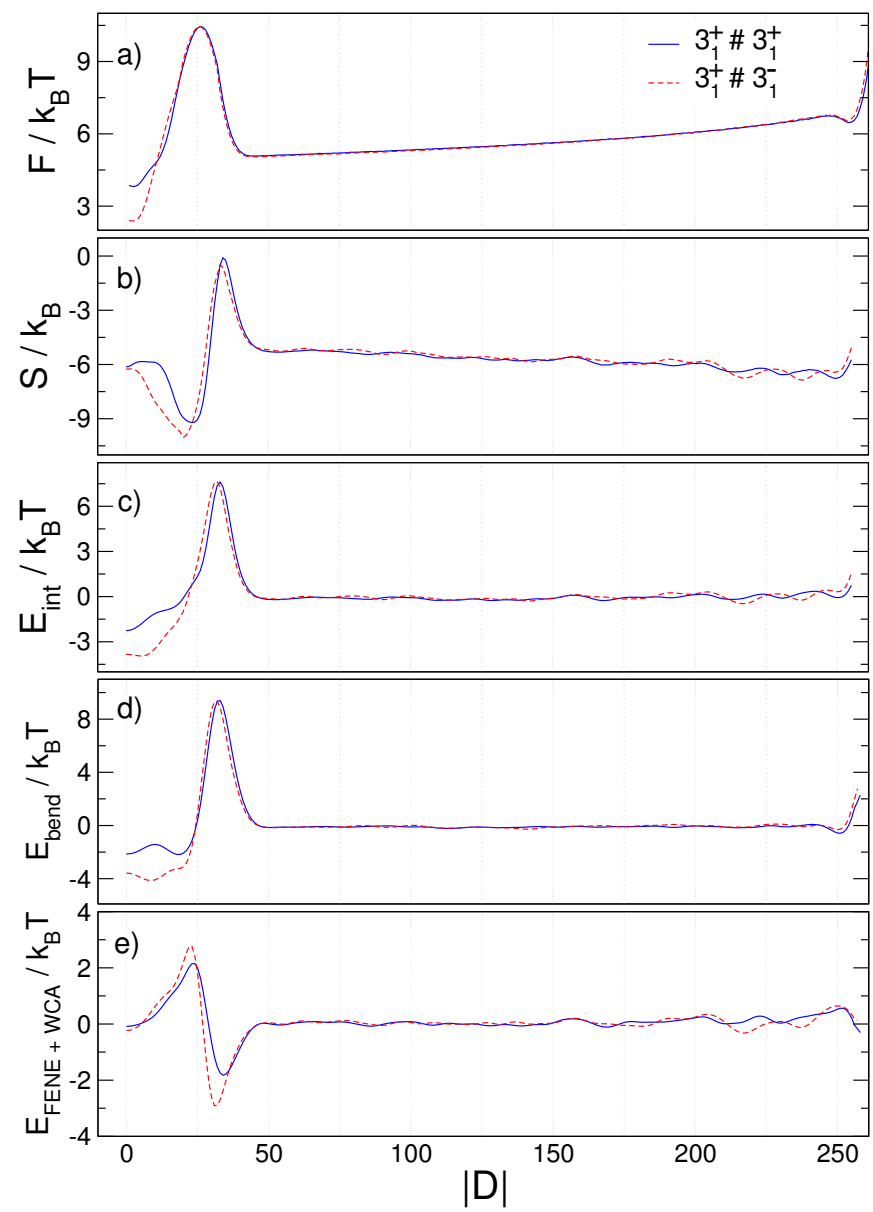

FIG. 4. Free energy of the $3_{1} 3_{1}$ system with same and opposite relative chirality, as a function of the separation $D$ between the two knots, decomposed in their different energetic and entropic contributions. We report the free energy $F(D)$ (a), the entropy $S(\mathrm{~b})$, and the internal potential energy $E_{\text {int }}(\mathrm{c})$. The entropy is obtained through the relation $F=E_{\text {int }}-T S$. In panels (d) and (e) we show how the internal potential energy is distributed, respectively, between the bending term and the remaining two contributions, namely the FENE and WCA potentials. See the main text for a discussion of the results.

firm that the observed increase of $F$ with $|D|$ when the two knots are separated is purely entropic. Secondly, and more importantly, they show that the differences we observe in the free energies of the $(+-)$ and $(++)$ systems originate from a complex interplay of internal energy and entropy. Specifically, the entropic contribution is higher for the $(++)$ case but is not high enough to overcome the energetic contribution favoring the $(+-)$ knot. The potential energy can be further decomposed into its main components: steric hindrance, bond extension, and bending energy. The data presented in Fig. 4 $4 \mathrm{~d}$-e clearly show that while all other energetic contributions are similar, the bending energies of the $(+-)$ and $(++)$ systems differ significantly at the position of the minimum of $F(D)$ 
by the same amount, $\sim 1.5 k_{B} T$. The same qualitative result holds also for the $3_{1}^{ \pm} 5_{1}^{-}$topologies (data not shown).

Given the observed competition between bending energy and entropy in the intertwined state, with the $(+-)$ system showing a lower bending energy but losing more entropy than the $(++)$ system, it is tempting to ascribe the difference in their free energy profile to a significantly different arrangement of the nested knot within the hosting knot in the two setups. However, further analyses and simulations are required to elucidate the exact mechanics underlying the chiral contribution, which is the object of an ongoing study.

Summing up, we have shown that a double knotted semiflexible polymer chain under strong stretching possesses a free energy minimum when the two knots are intertwined, showing that relatively tight knots can still pass through each other, and also that the depth of this minimum depends on the relative chirality of the two knots. In order to observe this effect, both knots must be chiral, with the knots of opposite chirality displaying a higher preference to stay intertwined. Furthermore, we showed that the major player in the chirality effect is the bending energy of the chain, which, we recall, is set to the characteristic persistence length of dsDNA.

The question then emerges, as to what are the specific features of the bending energy that would engender a chirality effect in knot interaction, and whether the stretching of the chain enters in some way as a significant constraint. If the chirality effect, described above, turns out to be robust and observable in a broad range of parameters, one can speculate further as to its importance in particular for chiral polymers such as dsDNA.

\section{ACKNOWLEDGMENTS}

L. Tubiana and R. Podgornik acknowledge support from the Slovenian Agency for Research and Development (ARRS grant No J1-4134). L. Tubiana acknowledges also support from the Mahlke-Oberman Stiftung and the European Union's Seventh Framework Programme for research, technological development and demonstration (grant No 609431). S. Najafi and R. Potestio are thankful to M. Heidari and R. Menichetti for an attentive reading of the manuscript and useful comments.
[1] M. Delbrück, in Mathematical problems in biological sciences, Proc. Symp. Appl. Math, Vol. 14, edited by R. E. Bellman (1962) p. 55.

[2] E. Frisch, H.L .and Wasserman, Journal of the American Chemical Society 83, 3789 (1961).

[3] D. Sumners and S. Whittington, Journal of Physics A: Mathematical and General 21, 1689 (1988).

[4] J. Arsuaga, M. Vazquez, S. Trigueros, D. W. Sumners, and J. Roca, Proceedings of the National Academy of Sciences 99, 5373 (2002).

[5] J. Arsuaga, M. Vazquez, P. McGuirk, S. Trigueros, D. W. Sumners, and J. Roca, Proceedings of the National Academy of Sciences of the United States of America 102, 9165 (2005).

[6] D. Marenduzzo, E. Orlandini, A. Stasiak, D. W. Sumners, L. Tubiana, and C. Micheletti, Proceedings of the National Academy of Sciences 106, 22269 (2009).

[7] W. Taylor, Nature 406, 916 (2000).

[8] P. Virnau, L. A. Mirny, and M. Kardar, PLoS Comput Biol 2, e122 (2006).

[9] N. P. King, E. O. Yeates, and T. O. Yeates, Journal of Molecular Biology 373, 153 (2007).

[10] R. Potestio, C. Micheletti, and H. Orland, PLoS computational biology 6, e1000864 (2010).

[11] T. Škrbić, C. Micheletti, and P. Faccioli, PLoS computational biology 8, e1002504 (2012).

[12] S. a Beccara, T. Škrbić, R. Covino, C. Micheletti, and P. Faccioli, PLoS computational biology 9, e1003002 (2013).

[13] S. Najafi and R. Potestio, The Journal of Chemical Physics 143, 243121 (2015).

[14] Y. Arai, R. Yasuda, K. Akashi, Y. Harada, H. Miyata, T. Kinosita, and H. Itoh, Nature 399, 446 (1999).
[15] A. Bates and A. Maxwell, DNA Topology (Oxford Bioscience, 2005).

[16] D. Meluzzi, D. Smith, and G. Arya, Annual review of biophysics 39, 349 (2010).

[17] C. Micheletti, D. Marenduzzo, and E. Orlandini, Physics Reports 504, 1 (2011).

[18] C. Micheletti and E. Orlandini, Soft Matter 8, 10959 (2012).

[19] A. Rosa, M. Di Ventra, and C. Micheletti, Physical Review Letters 109, 118301 (2012).

[20] I. Coluzza, P. D. van Oostrum, B. Capone, E. Reimhult, and C. Dellago, Physical review letters 110, 075501 (2013).

[21] E. Ben-Naim, Z. A. Daya, P. Vorobieff, and R. E. Ecke, Phys. Rev. Lett. 86, 1414 (2001).

[22] P. Virnau, Y. Kantor, and M. Kardar, Journal of the American Chemical Society 127, 15102 (2005).

[23] L. Tubiana, A. Rosa, F. Fragiacomo, and C. Micheletti, Macromolecules 46, 3669 (2013).

[24] R. Matthews, A. Louis, and J. Yeomans, EPL (Europhysics Letters) 89, 20001 (2010).

[25] A. Vologodskii, Biophys. J. 90, 1594 (2006).

[26] L. Huang and D. E. Makarov, The Journal of Physical Chemistry A 111, 10338 (2007), pMID: 17637045.

[27] A. Y. Grosberg and Y. Rabin, Phys. Rev. Lett. 99, 217801 (2007).

[28] L. Dai, C. B. Renner, and P. S. Doyle, Macromolecules 47, 6135 (2014).

[29] L. Dai, C. B. Renner, and P. S. Doyle, Phys. Rev. Lett. 114, 037801 (2015).

[30] J. des Cloizeaux, J. Phys. Lett. 42, L433 (1981).

[31] N. T. Moore, R. C. Lua, and A. Y. Grosberg, Proceedings of the National Academy of Sciences of the United States of America 101, 13431 (2004). 
[32] E. Orlandini, A. L. Stella, and C. Vanderzande, Phys Rev E Stat Nonlin Soft Matter Phys 82, 050804 (2010).

[33] M. L. Mansfield and J. F. Douglas, J. Chem. Phys. 133, 044903 (2010).

[34] A. M. Saitta, P. D. Soper, E. Wasserman, and M. L. Klein, Nature 399, 46 (1999).

[35] A. Stasiak, V. Katritch, J. Bednar, D. Michoud, and J. Dubochet, Nature 384, 122 (1996).

[36] C. Weber, A. Stasiak, P. D. L. Rios, and G. Dietler, Biophys. J. 90, 3100 (2006).

[37] Y. Diao, N. Pippenger, and D. W. Sumners, Journal of knot theory and its ramifications 3, 419 (1994).

[38] O. Farago, Y. Kantor, and M. Kardar, Europhys. Lett. 60, 53 (2002).

[39] K. Millett, A. Dobay, and A. Stasiak, Macromolecules 38, 601 (2005).

[40] B. Marcone, E. Orlandini, A. Stella, and F. Zonta, Physical Review E 75, 041105 (2007).

[41] M. Baiesi, E. Orlandini, and A. L. Stella, Journal of Statistical Mechanics: Theory and Experiment, P06012 (2010).

[42] K. Tsurusaki and T. Deguchi, Journal of the Physical Society of Japan 64, 1506 (1995).

[43] R. Zandi, Y. Kantor, and M. Kardar, ARI Bull. ITU 53, 6 (2003).

[44] P. G. Dommersnes, Y. Kantor, and M. Kardar, Physical Review E 66, 031802 (2002).

[45] B. Trefz, J. Siebert, and P. Virnau, Proceedings of the National Academy of Sciences 111, 7948 (2014).

[46] L. Tubiana, Phys. Rev. E 89, 052602 (2014).

[47] M. Caraglio, C. Micheletti, and E. Orlandini, Phys. Rev. Lett. 115, 188301 (2015).

[48] G. S. Grest and K. Kremer, Phys. Rev. A 33, 3628 (1986).

[49] K. Kremer and G. S. Grest, The Journal of Chemical Physics 92 (1990).

[50] V. V. Rybenkov, N. R. Cozzarelli, and A. V. Vologodskii, Proceedings of the National Academy of Sciences of the United States of America 90, 5307 (1993).
[51] L. Tubiana, E. Orlandini, and C. Micheletti, Progress of Theoretical Physics Supplement 191, 192 (2011).

[52] K. Koniaris, and M. Muthukumar, Phys. Rev. Lett. 66, 2211 (1991).

[53] E. Orlandini, and S. G. Whittington, Rev. Mod. Phys. 79, 601 (2007).

[54] M. K. Shimamura, and T. Deguchi, Physics Lettter A 274, 184 (2000).

[55] C. Micheletti, and E. Orlandini, ACS Macro Letter 3, 876 (2014).

[56] P. Poier, C. N. Likos, and R. Matthews, Macromolecules 47, 3394 (2014).

[57] E. G. Kim, and M. L. Klein, Macromolecules 37, 1674 (2004).

[58] A. Rosa, E. Orlandini, L. Tubiana, and C. Micheletti, Macromolecules 44, 8668 (2011).

[59] G. Dadamo, and C. Micheletti, Macromolecules 48, 6337 (2015).

[60] L. H. Kauffman, Transactions of the American Mathematical Society 318, 417 (1990).

[61] We remark that the inability of the Alexander polynomial to distinguish different chiralities does not affect our results, since in our setups the chirality of the knots cannot change and is fixed by the starting configuration.

[62] The number of observed events in which the trefoil knot enters into or exits an intertwined state with the $5_{1}$ knot is 580 out of $\sim 7.6 \times 10^{10}$ time steps for the $5_{1}^{-} 3_{1}^{-}(--)$ pair, and 992 out of $\sim 7.5 \times 10^{10}$ time steps for the $5_{1}^{-} 3_{1}^{+}$ (+-) pair.

[63] For this topology we measure 1280 events in which the one knots enters or exits the other, over a total of $\sim$ $7.5 \times 10^{10}$ time steps.

[64] In the case of the (+-) topology, we observe 3720 events out of $\sim 8.0 \times 10^{10}$ time steps in which one trefoil enters or exits the other; for the $(--)$ topology we have 3108 such events out of $\sim 8.0 \times 10^{10}$ time steps. 